\title{
IMPROVEMENT OF THE GEOMETRICAL STABILITY OF THE LHC CRYODIPOLES WHEN BLOCKING THE CENTRAL SUPPORT POST
}

\author{
F. Seyvet, J.-B. Jeanneret, A. Poncet, D. Tommasini, J. Beauquis, E.D. Fernandez Cano, E.Wildner \\ CERN, Geneva, Switzerland
}

\section{Abstract}

The LHC will be equipped with 1232 horizontally curved 16 meter long super-conducting dipole magnets cooled at $1.9 \mathrm{~K}$, supported within their vacuum vessel by three Glass Fiber Resin Epoxy (GFRE) support posts. The two support posts at the dipole extremities were initially designed free to slide longitudinally with respect to the vacuum vessel and the central support post free to slide transversely. However the magnet shape did not retain the tight geometrical tolerances, of the order of fractions of $\mathrm{mm}$, imposed by machine aperture and magnetic corrector centering requirements. Thereafter a modification to the supporting system, removing the initial transversal degree of freedom of the lower flange of the central support post with respect to the vacuum vessel, was designed and implemented. This paper describes the design of the magnet/cryostat interface with and without blockage of the central support post, analyzes the additional mechanical loads related to the modification and reviews the experimental results with respect to the requirements for beam aperture and magnetic corrector centering.

\section{MAGNET/CRYOSTAT INTERFACE AND BASELINE DESIGN CHANGE}

The initial design objective of the magnet/cryostat interface was mainly to support the cold mass inside its cryostat. Initially, no transversal load was expected on the three support posts, and the central support was designed not to constrain the cold mass transversely. An isostatic supporting system was designed with sliding pad and key allowing free translation of the support posts onto the vacuum vessel so as to cope with relative thermal contractions due to cryogenic operation. The extremity support posts were free to slide longitudinally along the yaxis, and the central support was free to slide laterally along the x-axis; the schematic of the cold mass supporting system and translation degrees of freedom in the vacuum vessel is represented in Figure 1.

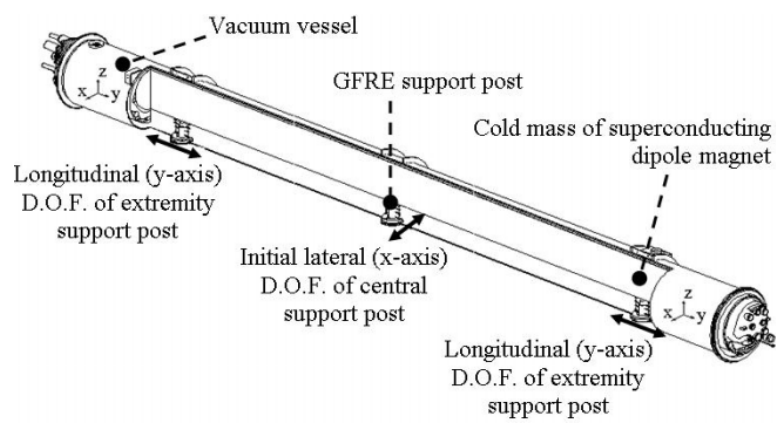

Figure 1: Cold mass/cryostat interface.
Because of demonstrated horizontal magnet geometry instabilities undermining the machine aperture of LHC [1], a modification to the supporting system was introduced. The aim was to constrain mechanically the lateral degree of freedom of the central support with respect to the vacuum vessel in order to stabilize the cold mass geometry [2]. As represented in Figure 2, a specific component was developed which allows blocking the lower flange of the support post with respect to the vacuum vessel, during the assembly of the cold mass within its cryostat at CERN. A key advantage of this design is that it can be retrofitted to the cryodipoles already assembled, without the need of de-cryostating.

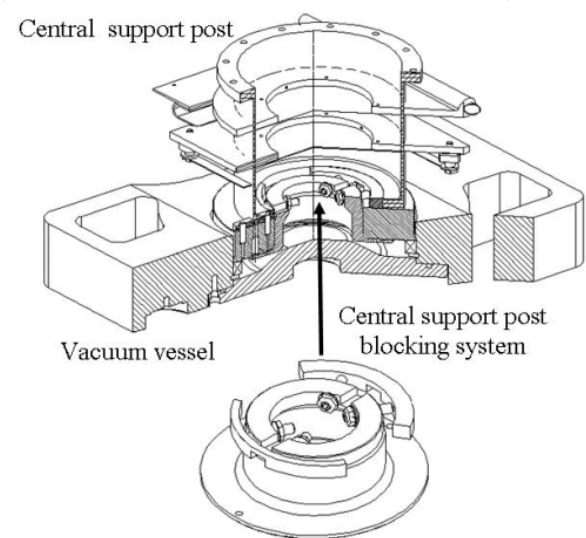

Figure 2: Cryodipole baseline design change by blocking the central support post.

A second key advantage of this design is to permit a tuning of the lateral $\mathrm{x}$-position of the lower flange of the central GFRE support post with respect to the vacuum vessel. This enables to adjust and control manually the horizontal geometry of the dipole cold mass.

\section{ADDITIONAL MECHANICAL LOADS}

The stabilization of the cold mass geometry and the adjustment of its shape generate additional mechanical loads in the magnet supporting system. The dipoles have a nominal horizontal sagitta of $9.14 \mathrm{~mm}$ over an active magnetic length of $14,343 \mathrm{~mm}$. The cold mass sagitta can be estimated using a best fit to a parabolic curve [3], from the data obtained during fiducialisation with a laser tracker, i.e. three dimensional measurements of the shape of the magnet beam tubes [4].

The dipole magnet sagitta display changes between manufacturing in industry and assembly within their cryostat at CERN. A quantification of this change has been established for 362 magnets, as shown in Figure 3. In case dipoles do not retain their initial horizontal geometry, the adjustment made at CERN via the blocking 
system is systematically done to restore the magnet initial shape for machine aperture and corrector magnets centering purposes.

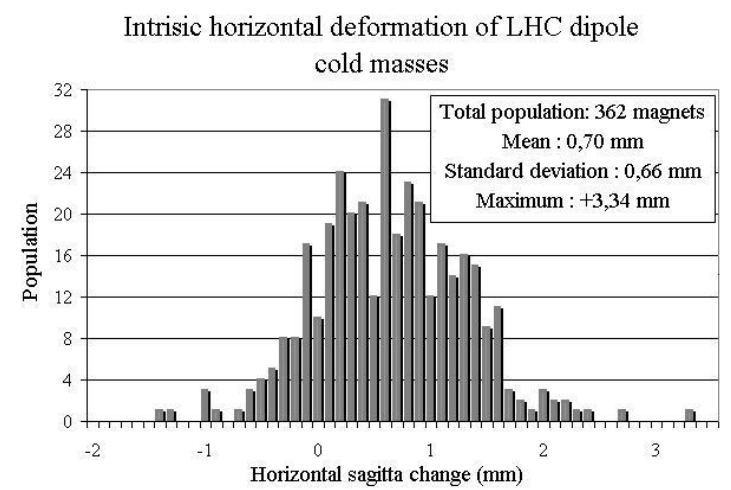

Figure 3: Magnet horizontal geometry changes between manufacture in industry and cryostat assembly at CERN.

The worst deformation observed within the analysed set of data is an increase of sagitta of $3.4 \mathrm{~mm}$, which corresponds to a distance from the mean value of the population 4.5 times larger than the standard deviation. Assuming a Gaussian distribution, the probability to have a magnet displaying such a geometric instability is less than $0.1 \%$ for the approximately 700 cryodipoles still to be assembled. This value is thus a reasonable maximum deformation to consider for additional lateral load determination. The dipole cold mass rigidity was determined via an experiment on magnet 1001 at CERN: its extremity feet were blocked in translation and the central foot of the cold mass was free to slide on a low friction bearings device. The rigidity of the cold mass was obtained by estimating the slope of the linear function relating the force to the displacement, see Figure 4, assuming that the cold mass behaves as a simply supported beam.

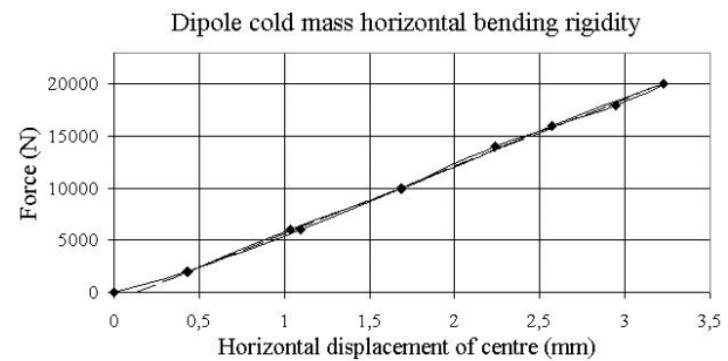

Figure 4: Measurements of the cold mass rigidity.
The rigidity of the cold mass, $E I$, has been estimated as $170+/-10 \mathrm{MN} . \mathrm{m} 2$, which is within $6 \%$ of previously published data [5]. Then, assuming that the cold mass behaves as a simply supported beam, given the distance between the cold mass end and the extremity support, $l$, and between the extremity support and the central support, $L / 2$, the additional lateral loads in the supporting system can be calculated as a function of the sagitta change of the cold mass, $d S$, as follows

$$
F=\frac{48 E I}{L^{2}(3 l+L)} \cdot d S
$$

where $F$ is the additional lateral load applied at the central support, and $F / 2$ the additional lateral load on the extremity supports. The parameters $l$ and $L$, given the geometry of the cold mass, are $1771.5 \mathrm{~mm}$ and $10.8 \mathrm{~m}$ respectively. Numerically, the additional loads related to the blocking of the central support are presented in Table 1 along with the baseline design operating loads. These latter ones were obtained previously [6] as a sum of the self weight loads, the effects of the LHC tunnel inclination, and the interconnections transversal misalignment loads. Six experiments on four magnets were performed to investigate the admissibility of the worst operating loads, considering both creep and rupture mechanisms. The experiments and their results are summarised in Table 1. For all tests, the vertical alignment of the magnet was set as it will be in the LHC machine. Tests with dipole 3004 [7] were performed with a configuration at the central support which was not representative of the production, test results are presented for information but were not considered for safety margins determination.

In Table 1, the minimum safety factors, obtained for the extremity supports, are 1.5 for creep and more than 1.9 for rupture. Even though these safety factors are relatively small, the occurrence probability of a sagitta deformation of $3.4 \mathrm{~mm}$ is extremely low, as mentioned previously. It can thus be safely assumed that the additional mechanical loads related to the blocking of the central support post are admissible for the operation of the LHC machine.

\section{GEOMETRY STABILITY ANALYSIS}

The stability of the assembled cryodipoles is assessed in comparing their horizontal geometry at different steps during their life cycle at CERN [2]. Additional fiducialisations of sets of magnets, with and without the design change implemented, were performed and

Table 1: Worst case operating loads on support posts and experimental validation.

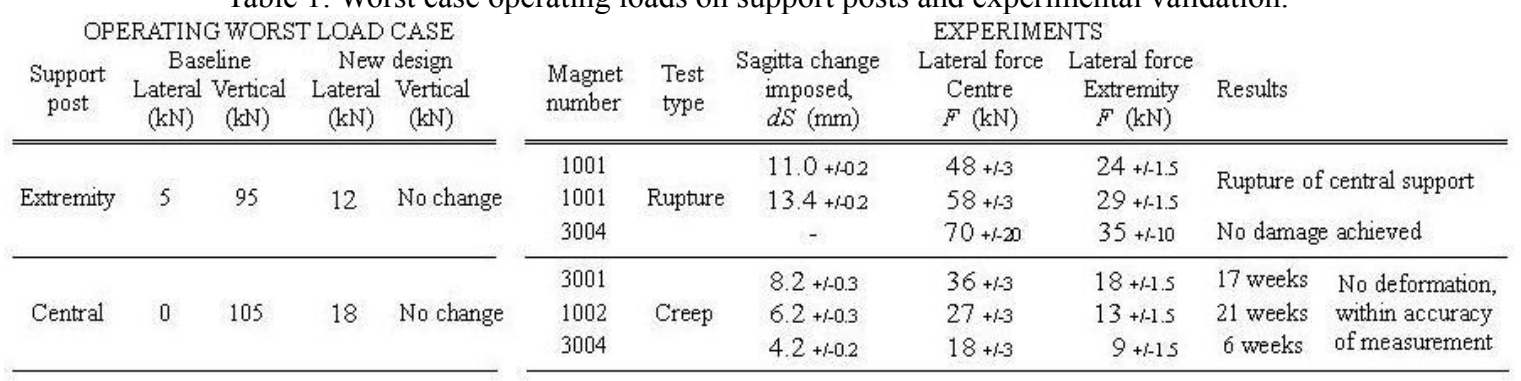


Two criteria have been defined in order to compare the magnets horizontal geometry between two stages, sagitta change and maximum local difference. The former one allows the global comparison of the overall horizontal geometry of the dipole cold mass. The latter one allows the local evaluation, at a given y-coordinate along the dipole, of the change of horizontal shape using $10^{\text {th }}$ order polynomial, superimposed at the extremity supports [3].

These data shall be considered with measurement errors. A single point, during a fiducialisation with a laser tracker, is measured with an accuracy of $0.15 \mathrm{~mm}$ r.m.s. The sagitta computation is also slightly affected by a bias introduced in the fit procedure, because the change of shape of the magnet is not necessarily an arc of a parabola. Thus, the overall error on the horizontal sagitta of the cryodipole certainly displays a standard deviation larger than $0.15 \mathrm{~mm}$ [8]. Similarly, the overall error for the maximum difference calculations displays a standard deviation that can reach $0.15 \mathrm{~mm}$.

Results are presented in Figure 5 and 6, along with the population size, $n$, the mean value, $\mu$, the standard deviation, $\sigma$, and the maximum absolute value, max. A significant improvement of the stability of the dipole cold masses with the blocking of the central foot is observed in Figure 5 and 6 . Furthermore, the statistical parameters, for the global and local magnet geometry changes, are all reduced by a minimum factor of 2.6 with the new design.

LHC dipole cold mass geometry stability Horizontal shape global change

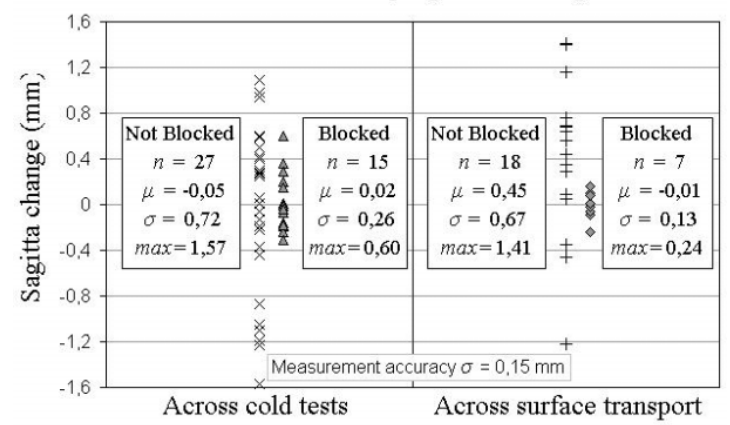

Figure 5: Global geometry stability analysis.

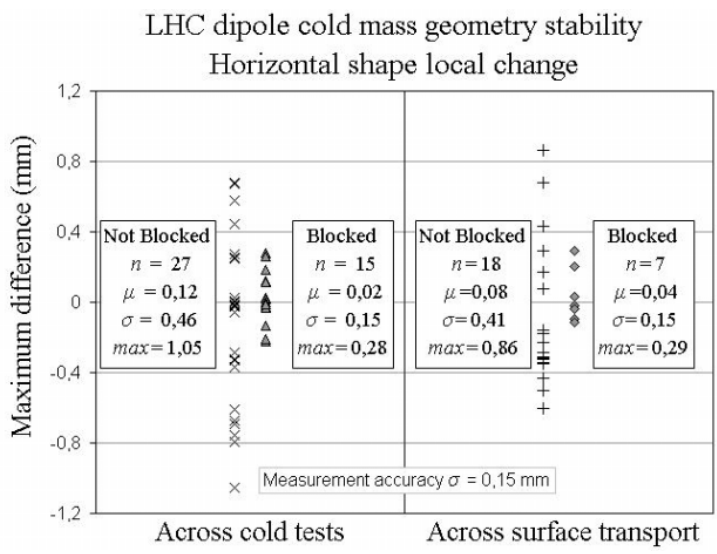

Figure 6: Local geometry stability analysis.
The effect of the cold mass deformations on the beam aperture and the positioning of the correctors magnets can be estimated. In both cases, the quantity to consider is of the order of half the change of the magnet sagitta. With the new design we get a value of approximately $0.4 \mathrm{~mm}$ when adding the maximum of cold tests and surface transport errors. This quantity is perfectly compatible with the break-up of the tolerances for LHC aperture and corrector magnet centering defined previously [9]. In comparison, for the baseline design, this value reached approximately $1.5 \mathrm{~mm}$, which is strongly out of the LHC machine specifications. The baseline design change of the cryodipole supporting system is thus fully justified and allows meeting the LHC initial requirements.

\section{CONCLUSIONS}

A modification to the baseline supporting system of the LHC dipole superconducting magnets, i.e. blocking the lower flange of the central support post with respect to the vacuum vessel, has been presented. The corresponding additional mechanical loads applied onto the GFRE support posts during machine operation were estimated and experimentally validated as admissible. The resulting stability improvement of the horizontal geometry of the magnets was shown to be significant, and the change to the baseline allows meeting the LHC initial beam aperture and magnetic corrector centering requirements.

This modification to the baseline supporting system of the LHC dipole superconducting magnets was implemented in production and retrofitted to the cryodipoles already assembled at CERN.

\section{REFERENCES}

[1] M. Bajko et al. "Executive summary of the workshop on LHC dipole geometry and stability" LHC Project Note 340, CERN, 2004.

[2] F. Seyvet et al. "Improvement of the stability of the horizontal geometry of LHC cryodipoles" LHC Project Note 359, CERN, 2004

[3] G. Gubello et al. "Shape Analysis of LHC preseries Dipoles” LHC Project Report 701, CERN, 2004

[4] D. Missiaen et al. "Fiducialisation of LHC cryodipoles" TS-SU Test Procedure EDMS 346576, CERN, 2002

[5] O. Calvet et al. "Analyse du comportement mécanique des cryodipôles du LHC - Statique" Note technique AT-CRI 2002-11, CERN, 2002

[6] V. Parma "Load carrying capacity of the LHC dipole supports" Private communication: presentation at the workshop on Main Dipole Geometry and Instability issues, CERN, 2004

[7] D. Simek "Dipole support post mechanical test" AT-CRI Test report EDMS 411325, CERN, 2003

[8] J. Beauquis et al. "LHC Dipole Geometry parameters, comparison between producers" 7th IWAA conference, Geneva, 2004

[9] See WGA site, http://lhc-proj-wga-wgr.web.cern.ch/ 